\title{
Stabilization of a Parallel Structured Single-link Flexible Arm Mounted on Moving Base Using Kalman Filter Based LEQG Control
}

\author{
Yuichi Sawada and Junki Kondo \\ Department of Mechanical and System Engineering, \\ Kyoto Institute of Technology \\ Matsugasaki Sakyo, Kyoto 606-8585, Japan \\ E-mail: sawada@kit.ac.jp
}

\begin{abstract}
In this paper, a method of risk-sensitive stabilization of a parallel structured single-link flexible arm using Kalman filter based LEQG control is presented. This arm is assumed to receive random disturbance which is generated by moving base such as vehicle bodies, mobile robots and so on. The random disturbance due to the moving base depends on its unknown acceleration which is assumed to be modeled by the white Gaussian noise. The structure of the arm is approximated by a single-link flexible arm consisting of an Euler-Bernoulli beam with the same boundary conditions of the parallel-structured one. The LEQG controller and the Kalman filter are constructed for the finite-dimensional model corresponding to the simplified structure model.
\end{abstract}

\section{Introduction}

Recently, the importance of flexible arms is increasing in various fields, because the flexible arms in operation have several advantages over rigid arms such as industrial robots. The advantages of the flexible arms are as follows: less energy consumption, less material, safety of human due to the mechanical flexibility and so forth. However, since the flexible arms are lightweight and low stiffness, they are easily affected by aerodynamic forces and/or external forces. Furthermore, in a case that the flexible arm is mounted on a vehicle or a mobile robot, the arm is affected by unknown external force due to the motion of the base. In such a case, we have to design a controller so that it suppresses the fluctuation of the tip of arm or the end-effecter.

In order to reduce the fluctuation of flexible arm mounted on the moving base for achieving high accurate operation, the random disturbances due to the aerodynamic force and the external force caused by the moving base should be suppressed. The LEQG control theory is suitable for such purpose. Optimal control problems of mechanical systems with random disturbance have been studied by many researchers. In many cases, the
LQG control theory has been employed for control the stochastic mechanical systems. On the other hand, the Linear-Exponential-Quadratic-Gaussian (LEQG) control is characterized by sensitive to large disturbance.

In this paper the LEQG control theory is applied to a parallel-structured single-link flexible arm which is very sensitive to the external disturbance such as the distributed random disturbance and the external force due to the moving base. The author has already studied the risk-sensitive control of flexible arms[1]. The LEQG control has been introduced by Jacobson, et al.[2] and [3], later, Whittle[4] formulated the risk-sensitive control problems, which are considered as the generalization of LEQG control problems. Besoussan[5] and Runolfsson[6] derived the risk-sensitive controller based on the partially observed data. This control theory has been used for some application fields, e.g., Financial engineering[7], missile guidance[8] and so forth.

This paper investigates the efficacy of the Kalman filter based LEQG control [9] for a parallel-structured single-link flexible arm mounted on a moving base. The feature of the parallel-structured single-link arm is that the arm holds sufficient rigidity along the vertical axis and mechanical flexibility along the displacement axis of the arm [10]. However, the exact dynamics of parallel-structured single-link flexible arm are described by quite complex nonlinear partial and ordinary differential equations, because the arm consists of two flexible beams which are disposed parallel. In this paper, such parallel-structured flexible arms are approximately modeled by a single-link flexible arm consisting of a flexible beam with the same boundary conditions as the parallel-structured one. The mathematical model of the flexible arm derived using the Hamilton's principle based on a simplified structure model of the parallel-structured flexible arm. The derived mathematical model is described by an Euler-Bernoulli type partial differential equation and ordinary differential equations. This mathematical model can be reduced to a set of ordinary differential equations using the modal expansion method. These are described by Itô stochastic differential equations. The controller is designed via 


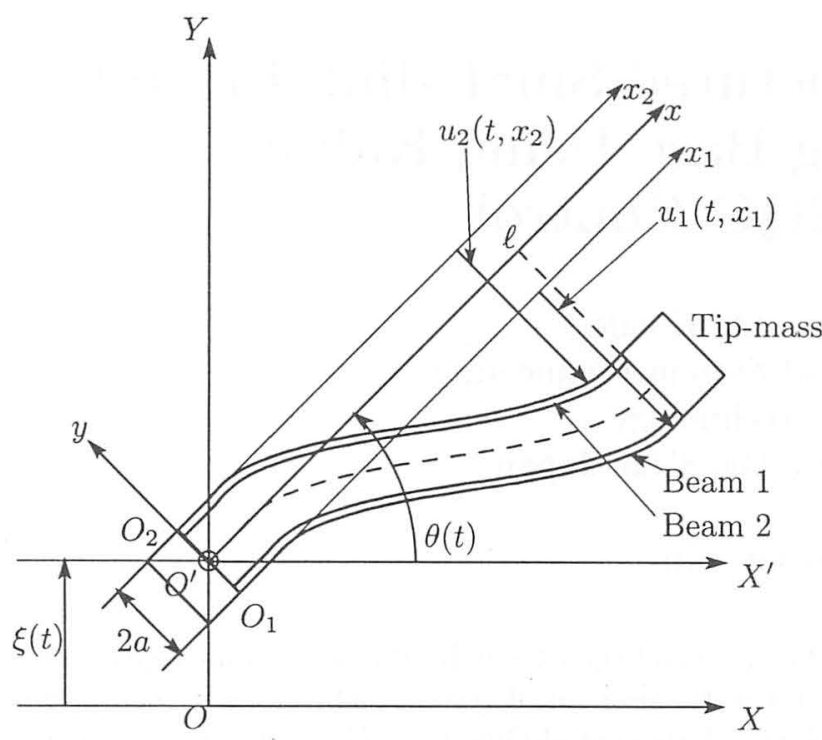

Fig. 1: Parallel-structured single-link flexible arm.

the LEQG control theory based on the partially observations which are measured by piezoelectric sensors and the potentiometer.

\section{Mathematical Model}

Consider a parallel-structured single-link flexible arm mounted on a moving base illustrated in Fig.1. This arm consists of two uniform Euler-Bernoulli beams with their length $\ell$. The end of each beam is clamped to the unit of hub and the other end is to a tip-mass.

Let $O X Y$ be the inertial Cartesian coordinate system; $O X^{\prime} Y$ the moving coordinate system for the moving base; Oxy the rotating coordinate system rotated by the servomotor installed at the hub; $O_{1} x_{1} y$ and $O_{2} x_{2} y$ the rotating coordinate systems for Beam 1 and 2 , respectively. $u_{i}\left(t, x_{i}\right)(i=1,2)$ denotes the displacement of Beam $i$ from the $x_{i}$-axis. Physical parameters of the beams are as follows: $\rho$ the uniform mass density; $S$ the cross section; $E I$ the uniform flexible rigidity (where $E$ denotes the Young's modulus and $I$ the second moment of cross sectional area); $m$ the mass of the tip-mass; and $c_{D}$ is the coefficient of Kelvin-Voigt type damping. The unit of hub has the moment of inertia $J_{0}$. The tip-mass has its mass $m$ and the moment of inertia $J_{1} . \xi(t)$ denotes the position of the moving base, which is the unknown function.

The exact mathematical model of the parallelstructured single-link flexible arm mounted on the moving base can be derived by the Hamilton's principle. However the exact mathematical model of the arm will be highly complex. In this work, for the sake of simplicity, the parallel-structured single-link flexible arm consisting of two Euler-Bernoulli type beams is mod-

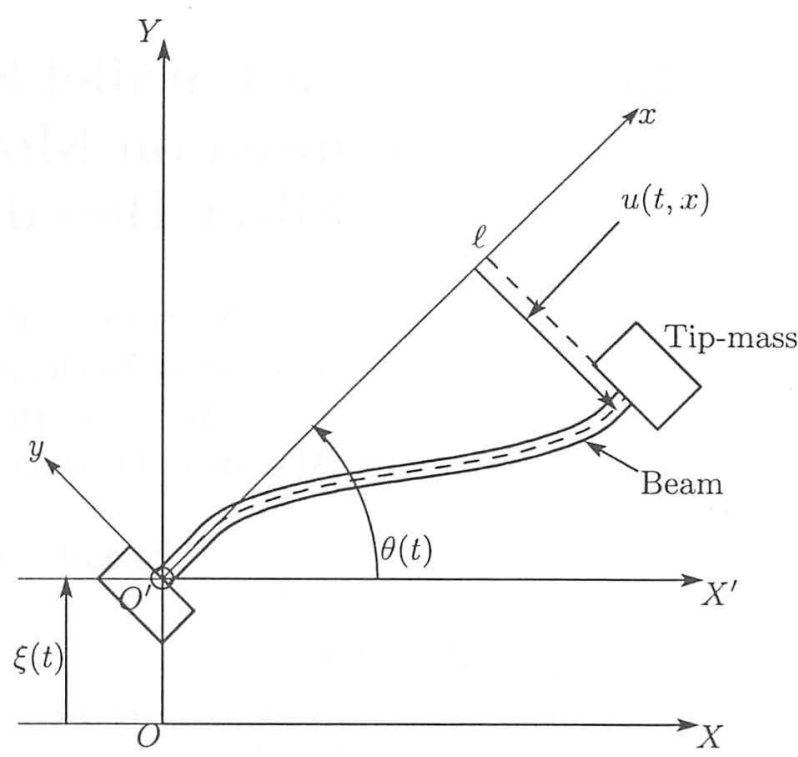

Fig. 2: Simplified structure of parallel-structured single-link flexible arm.

eled by a single-link flexible arm constructed by a flexible beam with the same boundary conditions as the parallel-structured one (see Fig.2).

$u(t, x)$ denotes the displacement of the approximated flexible arm from the equilibrium state of the beam; $\theta(t)$ the tangential axis of the root of the arm from the $X$-axis. In this paper, we assume that $|\theta(t)| \ll 1$ and $|u(t, x)|$ are sufficiently small.

Now we derive the mathematical model of the parallel-structured single-link flexible arm mounted on the moving base based on the simplified structure model of the arm using the Hamilton's principle. The position vectors of the arbitrary point of the beam, $r(t, x)$ and the center of mass of the tip-mass, $p(t)$, are expressed by the following vectors:

$$
\begin{gathered}
r(t, x)=\left[\begin{array}{c}
x \cos \theta(t)-\{u(t, x)-a\} \sin \theta(t) \\
x \sin \theta(t)+\{u(t, x)+a\} \sin \theta(t)+\xi(t)
\end{array}\right] \\
p(t)=\left[\begin{array}{c}
(\ell+h) \cos \theta(t)-u(t, \ell) \sin \theta(t) \\
(\ell+h) \sin \theta(t)+u(t, \ell) \sin \theta(t)+\xi(t)
\end{array}\right],
\end{gathered}
$$

where $2 h$ denotes the length of the tip-mass.

The kinetic energy of rigid part $T_{R}(t)$ can be described by the sum of the kinetic energies of translation and rotation of the tip-mass and the rotation of the hub attached to the shaft of the servomotor:

$$
T_{R}(t)=\frac{1}{2} m\|\dot{p}(t)\|^{2}+\frac{1}{2}\left(J_{0}+J_{1}\right) \dot{\theta}^{2}(t) .
$$

Similarly, the kinetic energy of the flexible beam is given 
by

$$
T_{F}(t)=\int_{0}^{\ell} \hat{T}(t, x) d x
$$

where $\hat{T}(t, x)=(1 / 2) \rho S\|\dot{r}(t, x)\|^{2}$. The total kinetic energy of the arm is

$$
T(t)=T_{R}(t)+T_{F}(t) .
$$

The potential energy of the whole arm is expressed by

$$
V(t)=\int_{0}^{\ell} \hat{V}(t, x) d x
$$

where $\hat{V}(t, x)=(1 / 2) E I\left\{\partial^{2} u(t, x) / \partial x^{2}\right\}$.

The Hamilton's principle is described by the following equation:

$$
\int_{t_{1}}^{t_{2}}\left\{\delta T(t)-\delta V(t)+\delta W_{n c}(t)\right\} d t=0,
$$

where $t_{1}$ and $t_{2}$ are arbitrary times; and $\delta W_{n c}(t)$ denotes the virtual work due to the nonconservative forces, e.g. internal damping forces of the beams, frictions, the control torque and external disturbances. $\delta W_{n c}(t)$ is expressed by

$$
\begin{array}{r}
\delta W_{n c}(t)=-\mu \dot{\theta}(t) \delta \theta+\tau(t) \delta \theta+g_{\theta} \gamma_{\theta}(t) \delta \theta \\
-\int_{0}^{\ell} c_{D}\left(\frac{\partial^{3} u(t, x)}{\partial x^{2} \partial t}\right) \delta u^{\prime \prime} d x \\
\quad+\int_{0}^{\ell} g_{f} \gamma(t, x) \delta u d x_{i},
\end{array}
$$

where the prime denotes the derivative with respect to $x ; \mu$ denotes the damping coefficient corresponding to the damping force acting at the shaft of the servomotor; $\tau(t)$ the control torque; $\gamma_{\theta}(t)$ the random disturbance acting at the rotation of the arm; $\gamma(t, x)$ the distributed random disturbance along the beam due to the aerodynamic resistance; and $g_{\theta}$ and $g_{f}$ are constants.

As the generalized coordinates, we consider the following variables: $\theta(t), \dot{\theta}(t), u(t, x), \dot{u}(t, x), u^{\prime}(t, x)$, $\dot{u}^{\prime}(t, x), u^{\prime \prime}(t, x), \dot{u}^{\prime \prime}(t, x), u(t, \ell), \dot{u}(t, \ell)$. Substituting $(7),(8)$ and (11) into (10); in addition, performing a large amount of calculations, we have the following nonlinear differential equations:

$$
\begin{aligned}
\rho S \frac{\partial^{2} u(t, x)}{\partial t^{2}}+c_{D} I \frac{\partial^{5} u(t, x)}{\partial t \partial x^{4}}+E I \frac{\partial^{4} u(t, x)}{\partial x^{4}} \\
=-\rho S x \ddot{\theta}(t)+\rho S \dot{\theta}^{2}(t) u(t, x)-\rho S \ddot{\xi}(t)+g_{f} \gamma(t, x) \\
-\left\{m(\ell+h) \ddot{\theta}(t)+m \frac{\partial^{2} u(t, x)}{\partial t^{2}}\right. \\
\left.-m \dot{\theta}^{2}(t) u(t, x)+m \ddot{\xi}(t)\right\} \delta(x-\ell)
\end{aligned}
$$

B.C.: $u(t, 0)=\frac{\partial u(t, 0)}{\partial x}=\frac{\partial u(t, \ell)}{\partial x}=\frac{\partial^{3} u(t, \ell)}{\partial x^{3}}=0$

$$
\begin{gathered}
\left\{J_{0}+J_{1}+m u^{2}(t, \ell)+m(\ell+h)^{2}+\rho S \int_{0}^{\ell} x^{2} d x\right\} \ddot{\theta}(t) \\
+\left\{\mu+\ell \dot{\xi}(t) \sin \theta(t)+2 \rho S \int_{0}^{\ell} u(t, x) \dot{u}(t, x) d x\right\} \dot{\theta}(t) \\
+m(\ell+h)\{\ddot{u}(t, \ell)+\ddot{\xi}(t) \cos \theta(t)-\dot{\xi}(t) \dot{\theta}(t) \sin \theta(t)\} \\
+\quad+2 m u(t, \ell) \dot{u}(t, \ell) \dot{\theta}(t) \\
+\rho S \int_{0}^{\ell}[x\{\ddot{u}(t, x)+\ddot{\xi}(t)\}+\ddot{\xi}(t) u(t, x) \sin \theta(t) \\
+\dot{\xi}(t) \dot{u}(t, x) \sin \theta(t)+\dot{\xi}(t) u(t, x) \dot{\theta}(t) \cos \theta(t)] d x \\
=\tau(t)+g_{\theta} \gamma_{\theta}(t), \quad(11)
\end{gathered}
$$

where $\delta(\cdot)$ denotes the Dirac's delta function. In this work, the acceleration of the moving base, i.e. $\ddot{\xi}(t)$, is assumed to be modeled by white Gaussian noise $\gamma_{\xi}(t)$, i.e., $\ddot{\xi}(t):=\gamma_{\xi}(t)$. The initial conditions of (9) and (11) are given by $u(0, x)=\dot{u}(0, x)=0$ and $\theta(0)=$ $\theta(0)=0$. In the future experiment of this work, since a velocity servomotor with a harmonic drive has been used, the dynamics of rotation can be approximated by the following equation (11):

$$
\ddot{\theta}(t)=f(t)+g_{\theta} \gamma_{\theta}(t)
$$

where $f(t)$ denotes the control input.

The observation is assumed to be obtained by means of $P$ strain sensors pasted on the surface of the beam at $x=\eta_{j},(j=1, \cdots, P)$ and a potentiometer installed at the shaft of the hub. The observations can be expressed by the following equations:

$$
\begin{aligned}
& y_{j}(t)=c_{j} \frac{\partial^{2} u\left(t, \eta_{j}\right)}{\partial x^{2}}+d_{j} \beta_{j}(t) \\
& y_{0}(t)=c_{0} \theta(t)+d_{0} \beta_{0}(t),
\end{aligned}
$$

where $y_{j}(t)(j=1, \cdots, P)$ represents the observation data of the $j$-th strain sensor; $y_{0}(t)$ the observation data of the potentiometer; $\beta_{0}(t)$ and $\beta_{j}(t)(j=0, \cdots, P)$ represent the observation noises modeled by the white Gaussian noise; $g_{j}$ and $d_{j}(j=0, \cdots, P)$ are constants.

The solution of (9) can be represented by

$$
u(t, x)=\sum_{k=1}^{N} u_{k}(t) \phi_{k}(x),
$$

where $N$ is a sufficiently large number; $\phi_{k}(x)(k=$ $1, \cdots, N)$ the $k$-th eigenfunction (mode function) corresponding to the eigenvalue $\lambda_{k}(k=1, \cdots, N)$ of the eigenvalue problem:

$$
\frac{E I}{\rho S} \frac{d^{4} \phi_{k}(x)}{d x^{4}}=\lambda_{k} \phi_{k}(x)
$$

with the boundary conditions $\left.\phi_{k}(0)=\phi_{k}^{\prime}(0)=\phi^{\prime} \ell\right)=$ $\phi_{k}^{\prime \prime \prime}(\ell)=0$ and $u_{k}(t)(k=1, \cdots, N)$ denotes the $k$-th modal displacement which is given by $u_{k}(t)=$ 
$\int_{0}^{\ell} u(t, x) \phi_{k}(x) d x$. The mode function $\phi_{k}(x) \quad(k=$ $1, \cdots, N)$ is the orthogonal function which holds the following inner product:

$$
\int_{0}^{\ell} \phi_{k}(x) \phi_{j}(x) d x= \begin{cases}1 & (k=j) \\ 0 & (k \neq j) .\end{cases}
$$

Substituting (15) with the property (17) into (9), we have the following modal representation model:

$$
\begin{aligned}
\sum_{j=1}^{N}\{ & \left.\delta_{k j}+\frac{m}{\rho S} \phi_{k}(\ell) \phi_{j}(\ell)\right\} \ddot{u}_{k}(t)+\frac{c_{D}}{E} \lambda_{k} \dot{u}_{k}(t)+\lambda_{k} u_{k}(t) \\
& =-\left\{\frac{m}{\rho S}(\ell+h) \phi_{k}(\ell)+\int_{0}^{\ell} x \phi_{k}(x) d x\right\} \ddot{\theta}(t) \\
- & \left\{\frac{m}{\rho S} \phi_{k}(\ell)+\int_{0}^{\ell} \phi_{k}(x) d x\right\} \ddot{\xi}(t)+\frac{g_{f}}{\rho S} \gamma_{k}(t),
\end{aligned}
$$

where $\delta_{j k}$ denotes the Kronecker delta; $\gamma_{k}(t)$ represents the random disturbance in modal representation corresponding to the distributed random disturbance $\gamma(t, x)$, which is given by $\gamma_{k}(t)=\int_{0}^{\ell} \gamma(t, x) \phi_{k}(x) d x$.

In this paper, since the motion of the base, $\xi(t)$, is assumed to be unknown, its' acceleration, $\ddot{\xi}(t)$, is assumed to be modeled by the white Gaussian noise, i.e. $\ddot{\xi}(t):=$ $\gamma_{\xi}(t)$, where $\gamma_{\xi}(t)$ denotes the white Gaussian noise. Let us define the state vector $v(t):=\left[u_{1}(t), u_{2}(t)\right.$, $\left.\cdots, u_{N}(t), \dot{u}_{1}(t), \dot{u}_{2}(t), \cdots, \dot{u}_{N}(t), \theta(t), \dot{\theta}(t)\right]^{\mathrm{T}}$. We obtain the following Ito stochastic state space model:

$$
\begin{aligned}
& d v(t)=A_{c} v(t) d t+b_{c} f(t) d t+G_{c} d w(t) \\
& d y(t)=C_{c} v(t) d t+E_{c} d \eta(t),
\end{aligned}
$$

where $y(t)$ represents the observation vector defined by $y(t)=\left[y_{0}(t), y_{1}(t), \cdots, y_{P}(t)\right]^{\mathrm{T}} ; w(t)=\left[w_{1}(t), \cdots\right.$, $\left.w_{N}(t), w_{\theta}(t), w_{\xi}(t)\right]^{\mathrm{T}} ; w_{k}(t)=\int_{0}^{t} \gamma_{k}(\tau) d \tau ; \gamma_{k}(t)=$ $\int_{0}^{\ell} \gamma(t, x) d x ; w_{\theta}(t)=\int_{0}^{t} \gamma_{\theta}(\tau) d \tau ; w_{\xi}(t)=\int_{0}^{t} \gamma_{\xi}(\tau) d \tau ;$ $\eta(t)=\int_{0}^{t} \beta(\tau) d \tau ; \beta(t)=\left[\beta_{1}(t), \cdots, \beta_{P}(t), \beta_{0}(t)\right]^{\mathrm{T}} ;$ $\mathcal{E}\left\{d w(t) d w^{\mathrm{T}}(t)\right\}=W d t ; \mathcal{E}\left\{d \eta(t) d \eta^{\mathrm{T}}(t)\right\}=V d t(\mathcal{E}\{\cdot\}:$ mathematical expectation); and

$$
A_{c}=M^{-1} A_{0}, b_{c}=M^{-1} b_{0}, G_{c}=M^{-1} G_{0}
$$$$
M=\left[\begin{array}{ccc}
I_{N} & 0 & 0 \\
0 & M_{22} & 0 \\
0 & 0 & I_{2}
\end{array}\right], G_{0}=\left[\begin{array}{ccc}
0 & 0 & 0 \\
\frac{g_{f}}{\rho S} I_{N} & g_{\theta} b_{2} & G_{23} \\
0 & G_{32} & 0
\end{array}\right]
$$$$
b_{0}=\left[\begin{array}{c}
0 \\
b_{1} \\
b_{2}
\end{array}\right] A_{0}=\left[\begin{array}{ccc}
0 & I_{N} & 0 \\
-\Lambda_{N} & -\frac{c_{D}}{E} \Lambda_{N} & 0 \\
0 & 0 & A_{33}
\end{array}\right]
$$$$
C_{c}=\left[\begin{array}{ccccc}
c_{1} \phi_{1}\left(\xi_{1}\right) & \cdots & c_{1} \phi_{N}\left(\xi_{1}\right) & 0 & 0 \\
\vdots & \ddots & \vdots & \vdots & \vdots \\
c_{P} \phi_{1}\left(\xi_{P}\right) & \cdots & c_{P} \phi_{N}\left(\xi_{P}\right) & 0 & 0 \\
0 & \cdots & 0 & 0 & c_{0}
\end{array}\right]
$$

$$
\begin{aligned}
& E_{c}=\operatorname{diag}\left\{e_{1}, \cdots, e_{P}, e_{0}\right\}, \Lambda_{N}=\operatorname{diag}\left\{\lambda_{1}, \cdots, \lambda_{N}\right\} \\
& A_{33}=\left[\begin{array}{ll}
0 & 0 \\
0 & 1
\end{array}\right], b_{1}=\left[\begin{array}{c}
\tilde{\phi}_{1} \\
\vdots \\
\tilde{\phi}_{N}
\end{array}\right], b_{2}=\left[\begin{array}{l}
0 \\
1
\end{array}\right]
\end{aligned}
$$$$
G_{23}=\left[\begin{array}{c}
\psi_{1} \\
\vdots \\
\psi_{N}
\end{array}\right], G_{32}=\left[\begin{array}{c}
0 \\
g_{\theta}
\end{array}\right]
$$$$
\tilde{\phi}_{i}=-\frac{m}{\rho S}(\ell+h) \phi_{i}(\ell)-\int_{0}^{\ell} x \phi_{i}(x) d x
$$$$
\psi_{i}=-\frac{m}{\rho S} \phi_{i}(\ell)-\int_{0}^{\ell} \phi_{i}(x) d x
$$

\section{LEQG Control}

In this section, an infinite horizon LEQG control problem of the parallel-structured single-link flexible arm is considered. The objective of the LEQG control is to suppress the random vibration of the arm excited by the distributed random disturbance and the external force due to the unknown motion of the moving base and to stabilize the angle position of the arm. For the parallel-structured single-link flexible arm, the fullstate information cannot directly be obtained by using the finite number of sensors by reason of the infinitedimensional system. In order to obtain the state estimate, a Kalman filter is employed by applying the separation theory. The Kalman filter is given by

$$
d \hat{v}(t)=A_{c} \hat{v}(t) d t+b_{c} f(t) d t+K_{c}\left\{d y(t)-C_{c} \hat{v}(t) d t\right\},
$$

where $K_{c}$ denotes the Kalman gain matrix defined by

$$
K_{c}=P_{c} C_{c}^{\mathrm{T}} V^{-1}
$$

and $P_{c}$ is the solution of the following algebraic Riccati equation:

$$
0=A_{c} P_{c}+P_{c} A_{c}^{\mathrm{T}}+G_{c} W G_{c}^{\mathrm{T}}-P_{c} C_{c}^{\mathrm{T}} V^{-1} C_{c} P_{c} .
$$

The cost functional to be minimized by the LEQG controller is described by

$$
J(f)=\lim _{T \rightarrow \infty} \frac{1}{T} \mathcal{E}\left\{\sigma \exp \left\{\frac{\sigma}{2} \int_{0}^{T} \Psi(t) d t\right\}\right\},
$$

where $\sigma$ denotes the risk-sensitive parameter which is a constant. The function $\Psi(t)$ is defined by the following quadratic form in the distributed parameter representation:

$$
\begin{aligned}
\Psi(t)= & q_{1} \ddot{u}^{2}(t, \ell)+\int_{0}^{\ell}\left[q_{2}\left\{u^{\prime \prime}(t, x)\right\}^{2}+q_{3}\left\{\dot{u}^{\prime \prime}(t, x)\right\}^{2}\right. \\
& \left.+q_{4} u^{2}(t, x)+q_{5} \dot{u}^{2}(t, x)\right] d x+q_{6} \ddot{\theta}^{2}(t) \\
& +q_{7} \dot{\theta}^{2}(t)+q_{8} \theta^{2}(t)+r_{f} f^{2}(t)
\end{aligned}
$$


Table 1: Physical parameters of the simplified structure of the flexible arm.

\begin{tabular}{cl}
\hline Parameters & \\
\hline$\ell$ & $0.25[\mathrm{~m}]$ \\
$E$ & $1.1 \times 10^{11}[\mathrm{~Pa}]$ \\
$S$ & $4.0 \times 10^{-5}\left[\mathrm{~m}^{2}\right]$ \\
$I$ & $3.3 \times 10^{-12}\left[\mathrm{~m}^{-4}\right]$ \\
$\rho$ & $8.8 \times 10^{3}\left[\mathrm{~kg} / \mathrm{m}^{3}\right]$ \\
$c_{D}$ & $4.82 \times 10^{7}\left[\mathrm{~N} \cdot \mathrm{s} / \mathrm{m}^{2}\right]$ \\
$m$ & $0.61[\mathrm{~kg}]$ \\
$h$ & $0.026[\mathrm{~m}]$ \\
\hline
\end{tabular}

where $q_{*}(*=1, \cdots, 8)$ are positive constants; $r_{f}$ a positive constant. Introducing the state vector $v(t)$ into (24), it is given by

$$
\begin{aligned}
J(f)= & \lim _{T \rightarrow \infty} \frac{1}{T} \mathcal{E}\left\{\sigma \operatorname { e x p } \left\{\frac{\sigma}{2} \int_{t_{0}}^{T} \hat{v}^{\mathrm{T}}(t) Q \hat{v}(t)\right.\right. \\
& \left.\left.+r_{f} f^{2}(t) d t\right\}\right\},
\end{aligned}
$$

where $\hat{v}(t)$ denotes the estimate of the state vector $v(t)$ given by the Kalman filter; $Q$ denotes the nonnegative definite symmetric matrix. The optimal control $f^{o}(t)$ based on the Kalman filter is given via the LEQG control theory [2] by

$$
f^{o}(t)=-r_{f}^{-1} b^{\mathrm{T}} S \hat{v}(t),
$$

where $S$ denotes the solution of the following algebraic Riccati equation:

$$
S A_{c}+A_{c} S-S\left(b r_{f}^{-1} b^{\mathrm{T}}-\sigma K_{c} V K_{c}^{\mathrm{T}}\right) S=0,
$$

where $K_{c}$ is the Kalman filter gain matrix.

\section{Numerical Simulation}

In the simulation study, we employed the simplified structure model of the parallel-structured singlelink flexible arm as a numerical model for the simulation. The simplified structure model of the parallelstructured single-link flexible arm is assumed to be made with the phosphor bronze with the length $\ell=$ $0.25[\mathrm{~m}]$, the thickness $1 \times 10^{-3}[\mathrm{~m}]$ and the width $2 \times$ $10^{-2}[\mathrm{~m}]$. The physical parameters of the flexible arm and the coefficients were listed in Table 1.

The observations are assumed to be measured by a piezoelectric film sensor with the length $b_{s}=3 \times$ $10^{-2}[\mathrm{~m}]$, width $1.2 \times 10^{-2}[\mathrm{~m}]$ pasted at $\eta_{1}=3 \times 10^{-3}[\mathrm{~m}]$ and a potentiometer installed at the hub. The parameters of the observation system were set as $c_{0}=10$, $c_{1}=-21.78, e_{0}=0.1$ and $e_{1}=0.01$. The covariance matrices for the system and observation noises were given by $W=\operatorname{diag}\{1,1,1\}$ and $V=\operatorname{diag}\{1,1\}$. The

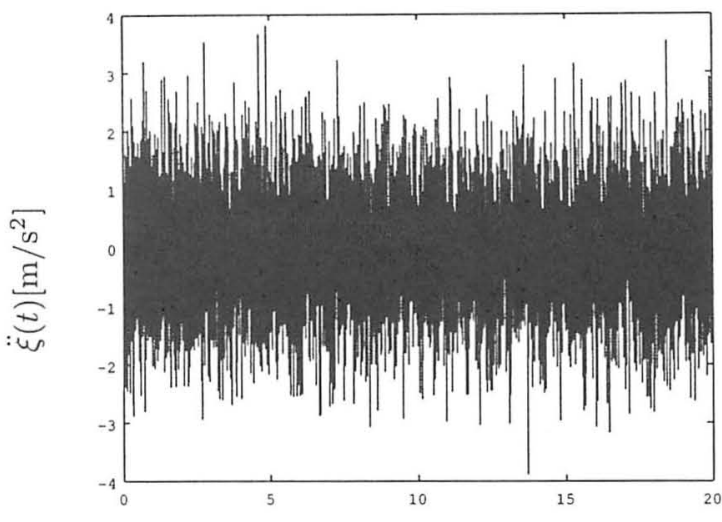

Time $t[\mathrm{~s}]$

Fig. 3: Acceleration of the moving base $\ddot{\xi}(t)$.

other coefficients are $g_{1}=1.0$ and $g_{2}=0.5$. The weight coefficients for controller were set as $q_{1}=2000, q_{2}=10$, $q_{3}=100, q_{4}=5000, q_{5}=10, q_{6}=10, q_{7}=40000$, $q_{8}=10, r_{f}=0.01$ and $\sigma=3.735 \times 10^{-5}$. The initial conditions of the flexible arm was $u(t, 0)=0[\mathrm{~m}]$, $\dot{u}(t, 0)=0[\mathrm{~m} / \mathrm{s}], \theta(0)=0[\mathrm{rad}]$ and $\dot{\theta}(0)=0[\mathrm{rad} / \mathrm{s}]$. The number of modes of the system was set as $N=2$. The time partition in numerical simulation was set as $\Delta t=1 \times 10^{-4}[\mathrm{~s}]$.

The numerical results are shown in Figs.4-6. Fig.3 depicts the acceleration of the moving base, $\ddot{\xi}(t)$, which is the white Gaussian noise. Fig.4 shows the sample run of the angle, $\theta(t)$. The angle amplitude of $\theta(t)$ was reduced after the controller was started at $t=5[\mathrm{~s}]$. Fig. 5 shows that the displacement of the tip-mass, $u(t, \ell)$. The displacement of the tip mass was computed by the following equation:

$$
u(t, \ell)=\sum_{k=1}^{2} u_{k}(t) \phi_{k}(\ell) .
$$

The displacement of tip-mass shows the small change after the controller was started. Fig.6 depicts the behavior of the control input $f^{o}(t)$.

\section{Conclusions}

In this paper the Kalman filter based LEQG control problem of a parallel-structured single-link flexible arm mounted on the moving base have been investigated. The simulation results showed that the LEQG controller suppressed well the random vibration of the arm mounted on the random moving base.

Acknowledgements: The authors wish to thank Mr. Toru Watanabe, Shimadzu corporation for his useful suggestions and advices. Part of this research is supported by the Ministry of Education, Culture, Sports, Science and Technology of Japan under the Grants-in- 


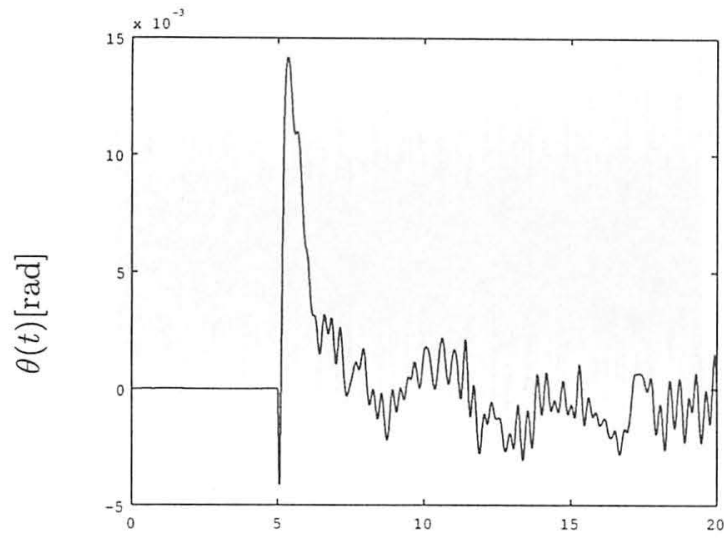

Time $t[\mathrm{~s}]$

Fig. 4: Sample run of angle of the flexible $\operatorname{arm} \theta(t)$ in the simulation study.

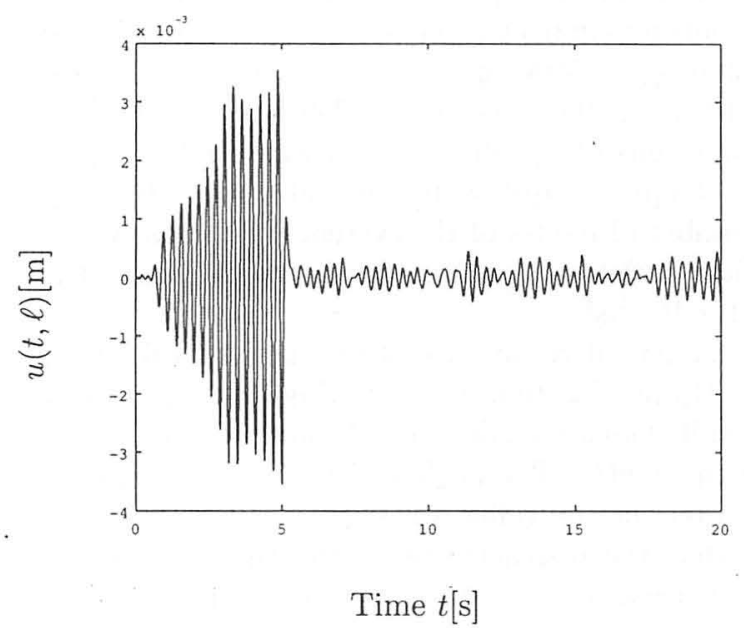

Fig. 5: Displacement of tip-mass, $u(t, \ell)$.

Aid for Scientific Research for Encouragement of the Grants-in-Aid for Scientific Research (C)-17560209.

\section{References}

[1] Y. Sawada and D. Koga, Risk-sensitive control of a single-link flexible arm, Proc. of the $43 \mathrm{rd}$ SICE Annual Conference 2004, Sapporo, pp.2337-2342, 2004.

[2] D. H. Jacobson, "Optimal Stochastic Linear Systems with Exponential Performance Criteria and Their Relation to Deterministic Differential Games," IEEE Trans. Automatic Control, vol. AC18, no. 2, pp. 124-131, 1973.

[3] J. L. Speyer, J. Deyst and D. H. Jacobson, Optimization of Stochastic Linear Systems with Additive Measurement and Process Noise Using Expo-

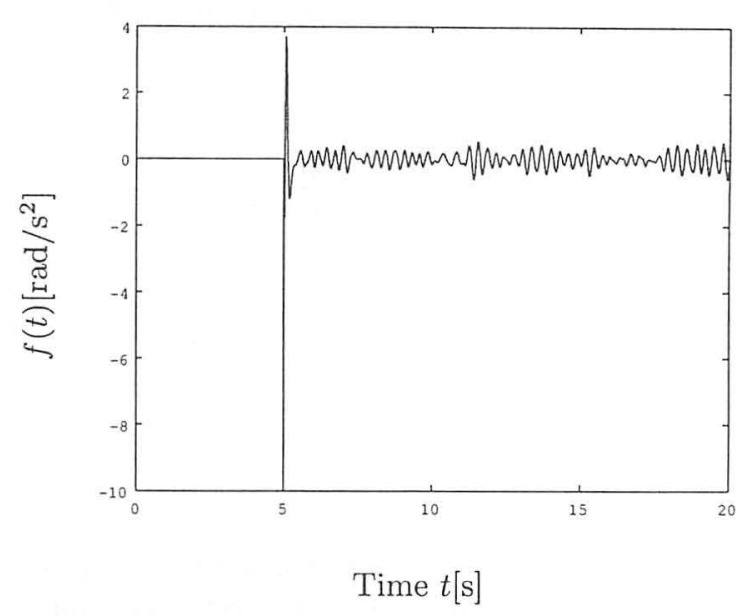

Fig. 6: Behaviors of the control input $f(t)$ in the simulation study.

nential Performance Criteria, IEEE Trans. Automatic Control, Vol.19, No.4, pp.358-366, 1974.

[4] P. Whittle, Risk-Sensitive Optimal Control, Wiley \& Sons, New York, 1990.

[5] A. Bensoussan and J. H. van Schuppen, Optimal control of partially observable stochastic system with an exponential-of-integralperformance index, SIAM J. Contr. Optim., vol.23, no.4, pp.599-613, 1985.

[6] T. Runolfsson, The equivalence between infinitehorizon optimal control of stochastic systems with exponential-of integral performance index and stochastic differential games, IEEE Trans. Automatic Control, vol.39, no.8, pp.1551-1563, 1994.

[7] H. Nagai, "Risk-Sensitive Stochastic Optimal Control and Mathematical Finance," J. Systems, Control and Information, vol. 44, no. 8, pp.447-454, 2000 (in Japanese).

[8] J. L. Speyer, "An Adaptive Terminal Guidance Scheme Based on an Exponential Cost Criterion with Application to Homing Missile Guidance," IEEE Trans. Automatic Control, vol. AC21, pp.371-375, 1976.

[9] J. M. Lin: System Design by Linear Exponential Quadratic Gaussian and Loop Transfer Recovery Methodology, J. Guidance, Control, and Dynamics, Vol.18, No.1, pp.189-192, 1995.

[10] Y. Sawada and T. Watanabe, "LQG Control of a Parallel-structured Single-link Flexible Arm," Proc. 51st Annual Conference of ISCIE, Kyoto, pp.372-373, 2007. 